ARTIGO ORIGINAL

\title{
Panorama das práticas de ecoinovação na indústria brasileira de revestimentos cerâmicos: 0 caso dos clusters de Criciúma e Santa Gertrudes
}

\author{
William Jeferson Vieira de Souza, Gabriela Scur, Wilson de Castro Hilsdorf \\ Centro Universitário FEI, Av. Humberto de Alencar Castelo Branco, 3972, CEP 09850-901, São Bernardo \\ do Campo, S.P., Brasil \\ E-mail: gabriela@fei.edu.br
}

\section{RESUMO}

Pesquisadores e empresas têm demonstrado um interesse crescente no tema da ecoinovação. Na indústria da cerâmica de revestimentos, vários impactos ambientais podem ser gerados em toda a cadeia produtiva. Este artigo tem como objetivo identificar como as práticas de ecoinovação são implementadas pelos produtores de revestimentos cerâmicos de Santa Gertrudes, estado de São Paulo, e Criciúma, estado de Santa Catarina no Brasil, clusters responsáveis por aproximadamente $80 \%$ da produção brasileira. Os resultados mostram que a maioria das ecoinovações implementadas é incremental e que as ecoinovações de processo e produto são consolidadas em ambos os clusters. Essa evidência mostra que os clusters enfrentam oportunidades inexploradas para estruturar ações organizacionais que podem ajudar as empresas a organizar seus esforços em direção à sustentabilidade. Outra constatação é que a implantação de ecoinovação nos clusters brasileiros é impulsionada principalmente por legislação específica e requisitos de mercado.

PALAVRAS CHAVE: Ecoinovação, cluster, práticas ambientais, cerâmica de revestimento, performance ambiental

\section{Introdução}

Bossle et al. (2016) destacam que inovação e sustentabilidade ambiental são conceitos centrais e ambos devem ser integrados às atividades de gestão e coordenação das empresas. Nesse contexto, a ecoinovação pode ser entendida como o desenvolvimento, assimilação ou exploração de um produto, serviço, processo produtivo ou método de gerenciamento, que é uma novidade radical ou incremental para uma organização. Quando comparado a alternativas pré-existentes, a ecoinovação apresenta melhores resultados, como por exemplo, redução dos riscos ambientais, riscos de poluição e impactos negativos relacionados ao uso dos recursos envolvidos (DÍAZ-GARCÍA; MORENO; MARTÍNEZ, 2015; ROSCOE; COUSINS; LAMING, 2016).

Algumas lacunas ainda trazem desafios para a construção de um entendimento pleno do tema da ecoinovação. Pouca atenção tem sido empregada na compreensão de como as empresas podem identificar e desenvolver ecoinovações (ROSCOE; COUSINS; LAMING, 2016). Além disso, um tópico de pesquisa em aberto é o modo como as empresas integram os conceitos de inovação e sustentabilidade nas atividades de negócios (BOSSLE et al., 2016). Díaz-García, Moreno e Martínez (2015) afirmam a necessidade do aumento no número de pesquisas relacionadas à ecoinovação em países recentemente industrializados ou em desenvolvimento, principalmente estudos de caso.

$\mathrm{Na}$ cadeia de revestimentos cerâmicos, diversos impactos ambientais podem ser gerados, desde a etapa de extração da matéria-prima até os processos de manufatura, comercialização, consumo, manutenção e 
instalação nas edificações (CPRH, 2010; BENVENISTE et al., 2014). No contexto brasileiro, os impactos ambientais estão relacionados à incidência de áreas degradadas, assoreamento e erosão das margens dos rios, desmatamento, emissões atmosféricas (gases poluentes e material particulado), alto consumo de energia, uso de material com substâncias tóxicas, ruído, vibração, emissão de líquidos poluentes, geração de resíduos sólidos e destinação inadequada de embalagens. (CETESB, 2008; CPRH, 2010).

Com base nos estudos anteriores sobre a importância da ecoinovação, este artigo discute como as ecoinovações são implementadas nos fabricantes brasileiros de revestimentos cerâmicos. Este artigo tem como objetivo identificar como as práticas de ecoinovação são implementadas em produtores de revestimentos cerâmicos de Santa Gertrudes, no estado de São Paulo, e Criciúma, no estado de Santa Catarina, clusters responsáveis por aproximadamente $80 \%$ da produção brasileira.

\section{Revisão de literatura}

\subsection{Práticas de ecoinovação de processo}

Carrillo-hermosilla, Río e Könnölä (2010) apontam que uma ecoinovação está relacionada à eficiência quando a otimização de subsistemas permite que uma organização produza uma quantidade maior de produtos, enquanto reduz a quantidade de recursos utilizados e os índices de poluição envolvidos. Diferentes indicadores de ecoeficiência são usados para medir os resultados de uma ecoinovação de processo (LEVIDOW et al., 2016). Por exemplo, os indicadores de ecoeficiência focados na produtividade são dados pela razão entre o resultado econômico (lucro ou quantidade de produtos produzidos) e o impacto ambiental associado (emissão de poluentes, geração de resíduos e consumo de recursos) (ARAMPATZIS et al., 2016). Por outro lado, os indicadores de ecoeficiência focados na intensidade da carga ambiental são dados pela razão entre a carga ambiental (emissão de poluentes, geração de resíduos e consumo de recursos) e a unidade de atividade econômica gerada (por exemplo, unidades monetárias ou unidade produzida). Stanchev e Ribarova (2016) destacam que as diretrizes de ecoeficiência são abordadas pela norma ISO 14045.

A literatura também relaciona a ecoinovação de processo com a produção mais limpa ( $\mathrm{P}+\mathrm{L})$ (KLEWITZ; HANSEN, 2014; LEVIDOW et al., 2016; ROSCOE; COUSINS; LAMING, 2016). A P+L é viabilizada por meio de tecnologias projetadas para garantir que a proteção ambiental seja parte integrante do processo de produção (DEMIREL; KESIDOU, 2011). Triguero, Moreno e Davia (2013) argumentam que as tecnologias mais limpas resultam na redução da quantidade total de resíduos gerados e no decréscimo da poluição préexistente, estes resultados são viabilizados por mudanças significativas em todo o processo de produção. Triguero, Moreno e Davia (2013) também vinculam a ecoinovação de processo às tecnologias de fim de tubo. O papel de uma ecoinovação ligada às tecnologias de fim de tubo é caracterizado como a incorporação de um componente que melhora a qualidade ambiental da organização (CARRILLO-HERMOSILLA; RÍO; KÖNNÖLÄ, 2010). A ecoinovação gerada minimiza os impactos ambientais negativos sem necessariamente alterar os processos e sistemas envolvidos (CARRILLO-HERMOSILLA; RÍO; KÖNNÖLÄ, 2010).

Faulkner e Badurdeen (2014) destacam o potencial da metodologia dos 6R's (reduzir, recuperar, reuso, reciclar, remanufaturar e redesign) para a geração de inovações de cunho sustentável nos processos de manufatura e nos fluxos de materiais envolvidos no ciclo de vida de um produto.

A adoção de processos ambientalmente responsáveis pode ser um pré-requisito ou um fator de potencialização para o advento de uma ecoinovação de produto ou para a melhoria de um produto preexistente com a possibilidade de afetar a cadeia de suprimentos como um todo (TRIGUERO; MORENO; DAVIA, 2013; KLEWITZ; HANSEN, 2014). 


\subsection{Práticas de ecoinovação de produto}

Roscoe, Cousins e Laming, (2016) sugerem que a ecoinovação de produto está relacionada ao design de um eco-produto que, quando comparado às alternativas existentes, gera uma quantidade menor de resíduos ou opera com energias renováveis. Nesse contexto, a ecoinovação do produto pode ser vinculada ao conceito de eco-design. Este é um termo consolidado nas comunidades acadêmica e empresarial, sendo abordado na norma ISO 14006 (BRONES; CARVALHO, 2015). Laosirihongthong, Adebanjo e Tan (2013), afirmam que as práticas de eco-design podem ser direcionadas ao produto ou embalagem. $\mathrm{O}$ foco no produto abrange um conjunto de ferramentas, métodos e princípios que ajudam os profissionais de design a incorporar o objetivo da redução de impacto ambiental nas atividades de desenvolvimento do produto. O foco na embalagem está relacionado ao conceito de embalagem ecológica, ou seja, a integração dos aspectos ambiental, econômico e social da sustentabilidade no desenvolvimento de embalagens com as melhores características de conservação do produto, capacidade de armazenamento, peso, facilidade de uso e composição de materiais recicláveis e/ou biodegradáveis (JIMÉNEZ-GUERRERO; ABAD; SANTAMARÍA, 2015).

Klewitz e Hansen (2014) destacam a avaliação do ciclo de vida (ACV) como uma prática de ecoinovação. A ACV tem diretrizes estabelecidas nas normas ISO 14040 e 14044, e, quantifica o impacto ambiental de um produto em cada etapa do ciclo de vida (JACQUEMIN; PONTALIER; SABLAYROLLES, 2012; POUDELET et al., 2012). Os indicadores de desempenho ambiental de um produto analisado com a ACV podem ser quantificados nos estágios de extração de matéria-prima, produção de materiais, fabricação de produtos, uso do produto, fim do ciclo de vida e transporte (ARENA; AZZONE; CONTE, 2013). Além disso, Klewitz e Hansen (2014) incluem o programa de rotulagem ecológica no contexto da inovação de produtos. Os princípios da rotulagem ecológica são abordados pela norma ISO 14020 (PRIETO-SANDOVAL et al., 2016).

\subsection{Práticas de ecoinovação organizacional}

A ecoinovação organizacional é relacionada ao desenvolvimento de novos métodos de gestão, focados na redução do impacto ambiental, na melhoria das condições de trabalho e no bem-estar dos funcionários (KLEWITZ; HANSEN, 2014; ROSCOE; COUSINS; LAMING, 2016; TRIGUERO; MORENO; DAVIA, 2013). Várias iniciativas podem resultar em ecoinovações organizacionais (CHENG; SHIU, 2012). Triguero, Moreno e Davia (2013) destacam o desenvolvimento de treinamentos ambientais para os funcionários. Klewitz e Hansen (2014) propõem a criação de um programa de compras com fornecedores locais para reduzir as emissões de poluentes relacionadas ao transporte. Outra iniciativa sugerida pelos autores é a implementação de estruturas organizacionais focadas no meio ambiente, como por exemplo, departamentos, equipes, comitês e unidades interdepartamentais. A literatura aponta o SGA como uma importante prática de ecoinovação organizacional, sendo frequentemente discutido como um meio para sistematicamente realizar a gestão das questões ambientais no que tange a mensuração, o reporte e as responsabilidades para lidar com o uso de recursos (materiais, energia e água) e os consequentes resíduos gerados (TRIGUERO; MORENO; DAVIA, 2013; KLEWITZ; HANSEN, 2014; BOSSLE et al., 2016). As principais diretrizes para certificação de um SGA são o sistema europeu de eco-gestão e auditorias ou eco-management and audit scheme (EMAS) e ISO 14001 (Sistemas de gestão ambiental - requisitos com orientações para uso) (LOZANO, 2012; CAMPOS et al., 2015).

Estudos anteriores destacam a utilização da classificação de indicadores de desempenho ambiental estabelecida na ISO 14031 (Avaliação de desempenho ambiental) para classificar os indicadores que podem ser atrelados a mensuração de um SGA (CAMPOS et al., 2015; NGUYEN; HENS, 2015). A classificação da ISO 14031 divide os indicadores de desempenho ambiental (IDA) nas categorias de indicadores de desempenho gerencial (IDG) e indicadores de desempenho operacional (IDO). Os indicadores de desempenho gerencial (IDG) provem informações acerca dos esforços gerenciais que influenciam 
positivamente a performance ambiental da organização como um todo (CAMPOS et al., 2015). Os indicadores de desempenho operacional (IDO) provem informações de desempenho ambiental relacionadas aos processos de operação de uma organização (CAMPOS et al., 2015).

Com base no conteúdo exposto, elaborou-se um modelo teórico (figura 1) para a análise da implantação da ecoinovação (em cada unidade de análise) que contempla: o mapeamento das práticas e métricas de ecoinovação de produto, processo e organizacional; e, a análise da categoria de mudança gerada por cada ecoinovação implantada.

\begin{tabular}{|c|c|c|}
\hline \multicolumn{3}{|c|}{ Mapeamento das práticas e métricas de ecoinovação implantadas } \\
\hline Processo & \multirow{5}{*}{ 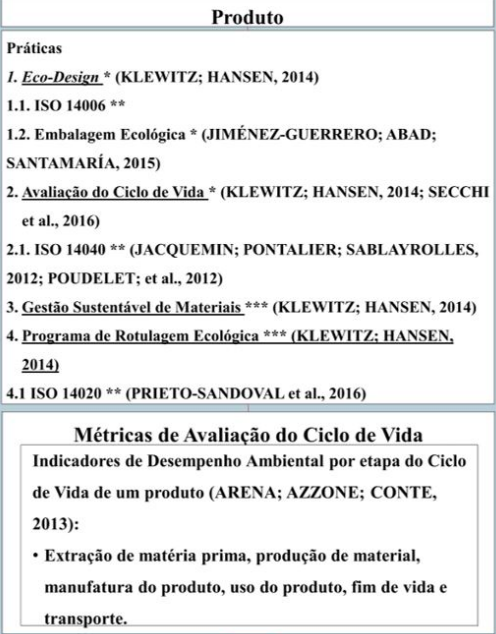 } & Organizacional \\
\hline 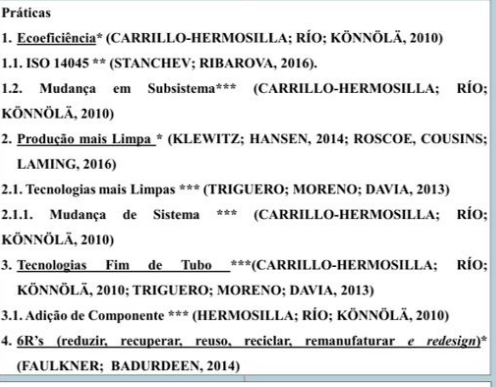 & & $\begin{array}{l}\text { Práticas } \\
\text { 1. Desenvolvimento de Treinamentos Ambientais } \\
\text { (TRIGUERO; MORENO; DAVIA, 2013) } \\
\text { 2. Programa de Compras com Fornecedores Locais *** } \\
\text { (KLEWITZ; HANSEN, 2014) } \\
\text { 3. Implantacão de Estruturas Organizacionais com foco } \\
\text { Ambiental *** (KLEWITZ; HANSEN, 2014) } \\
\text { 4. Sistema de Gestão Ambiental * (TRIGUERO; MORENO; } \\
\text { DAVIA, 2013; KLEWITZ; HANSEN, 2014; BOSSLE et al.. } \\
\text { 2016) } \\
\text { 4.1. ISO 14001 ** (LOZANO, 2012; CAMPOS et al, 2015) } \\
\text { 4.2. EMAS ** (LOZANO, 2012; CAMPOS et al., 2015) }\end{array}$ \\
\hline Métricas de Ecoeficiência & & Métricas do Sistema de Gestão Ambiental \\
\hline $\begin{array}{c}\text { Indicadores de Ecoeficiência focados na Produtividade } \\
\text { (ARAMPATZIS et al., 2016) } \\
\end{array}$ & & $\begin{array}{l}\text { Indicadores de Desempenho Gerencial } \\
\text { (CAMPOS et al., 2015; NGUYEN; HENS, 2015) }\end{array}$ \\
\hline $\begin{array}{l}\text { Indicadores de Ecoeficiência focados na Intensidade de } \\
\text { Carga Ambiental } \\
(\text { OECD, 2009) }\end{array}$ & & $\begin{array}{l}\text { Indicadores de Desempenho Operacional } \\
\text { (CAMPOS et al., 2015; NGUYEN; HENS, 2015) }\end{array}$ \\
\hline \multicolumn{3}{|c|}{2} \\
\hline \multicolumn{3}{|c|}{$\begin{array}{l}\text { Análise da categoria de mudança gerada por ecoinovação implantada } \\
\text { (CARRILLO-HERMOSILLA; RÍO; KÖNNÖLÄ, 2010; TRIGUERO; MORENO; DAVIA, 2013) }\end{array}$} \\
\hline Ecoinovação Incremental & & Ecoinovação Radical \\
\hline
\end{tabular}

Legenda: * Conceito, metodologia ou sistema / ** Padrão ou norma / *** Iniciativa de aplicação: mudanças, tecnologias, procedimentos, programas e estruturas

Fig. 1. Modelo teórico proposto para a análise da implantação das práticas de ecoinovação

\section{Procedimentos metodológicos}

Foram realizados múltiplos estudos de caso em 14 empresas visitadas, sendo 11 do cluster de Santa Gertrudes e 3 do cluster de Criciúma, nas quais 22 especialistas foram entrevistados.

Além desses entrevistados, buscou-se informações também com outros especialistas do setor, sendo dois acadêmicos na Universidade Federal de São Carlos, três representantes de associações de classe ligadas ao setor ceramista - CCB (Centro Cerâmico do Brasil) e ASPACER (Associação paulista das cerâmicas de revestimento), dois representantes de órgão governamental - CETESB (Companhia Ambiental do Estado de São Paulo) e um representante de fornecedor de esmaltes. Outros dados foram ainda obtidos por meio do website das empresas, de documentos coletados, observações diretas e participação em eventos e feiras do setor, o que permitiu a triangulação dos dados para a tabulação, combinação e análise dos resultados obtidos (EISENHARDT, 1989).

\section{Resultados}

\subsection{Práticas de ecoinovação de processo}

Após a aplicação das práticas de ecoinovação de produto os fabricantes de ambos os clusters, relataram o alcance dos seguintes resultados: redução no consumo de gás natural e emissão de fluoreto; mitigação da emissão de fluoreto; redução da emissão de material particulado; prevenção do descarte inadequado de 
efluentes líquidos industriais não tratados; redução no consumo de água de fonte externa; incorporação de resíduos sólidos (cal fluorada e lodo) dos sistemas de tratamento na massa cerâmica; mitigar os prejuízos dos desperdícios de matérias-primas; redução do consumo e desperdício de pallets; redução do consumo de pigmentos e insumos de condução; e, redução no consumo de tinta para impressão de embalagens. A única peculiaridade verificada foi o resultado da redução do consumo e do desperdício de esmaltes identificado apenas no cluster de Criciúma.

A recuperação da energia térmica está ligada à produção mais limpa, pois diminui a emissão de fluoreto. Outro benefício é a diminuição do custo de gás natural.

A maioria das empresas do cluster de Santa Gertrudes utilizam algum tipo de tecnologia de fim de tubo para o controle e mitigação da emissão dos poluentes fluoreto gerado na etapa de queima e material particulado gerado nas etapas de preparação da massa e processamento cerâmico. As principais ecoinovações utilizadas no cluster de Santa Gertrudes são o sistema de filtro manga e o lavador de gases. Isto decorre da necessidade de atendimento ao limite máximo de emissão $\left(30 \mathrm{mg} / \mathrm{Nm}^{3}\right.$ de material particulado e $\mathrm{mg} / \mathrm{Nm}^{3} \mathrm{a}$ $18 \%$ de $O_{2}$ ) fixado em regulamentação da CETESB.

De maneira similar aos produtores portugueses (ALMEIDA et al., 2016) e aos espanhóis (GABALDÓN-ESTEVAN; CRIADO; MONFORT, 2014), todas as organizações reaproveitam nos processos industriais o efluente líquido industrial tratado. Outro benefício é a redução do custo de extração da água de poço artesiano. Segundo os entrevistados a economia no consumo de água pode ser de aproximadamente $30 \%$.

Os produtores de Santa Gertrudes e Criciúma desenvolveram processos industriais para a dosagem dos resíduos sólidos da cal fluorada (gerada no filtro manga de mitigação da emissão de fluoreto) e do lodo (gerado na ETE) na composição da massa cerâmica.

As matérias-primas retidas nos filtros manga e os cacos queimados e também os não queimados, em ambos os clusters, são largamente reaproveitados na composição da massa cerâmica.

A instalação de impressão HD pode ser considerada uma tecnologia limpa, pois quando comparada à serigrafia, ela melhora a ecoeficiência, pois possibilita uma redução no consumo de pigmento e dos insumos de condução dos pigmentos.

\subsection{Práticas de ecoinovação de produto}

As inovações que agregaram atributos ambientais aos produtos viabilizaram em ambos os clusters o alcance dos seguintes resultados: reaproveitamento de resíduos sólidos; redução no consumo de matériasprimas, água e energias; redução no consumo de papelão; redução no consumo das tintas para impressão de embalagens; e, eliminação de substâncias tóxicas. Estes êxitos verificados nos dois clusters mostram uma uniformidade no modo que os produtores brasileiros mobilizam suas equipes de design para desenvolver ecoinovações que reduzam o consumo de recursos naturais e eliminem o uso de substâncias prejudiciais ao meio ambiente.

O resultado de redução na geração de resíduos sólidos foi evidenciado somente no cluster de Santa Gertrudes. Isto mostra que os produtores deste cluster empregam esforços adicionais no ecodesign de produtos para reduzir custos operacionais. Também pode ser destacado os resultados de desenvolvimento de novos produtos e de soluções para a comunicação dos atributos ambientais das placas cerâmicas, que mostram que o cluster de Criciúma tem um esforço particular para oferecer revestimentos que melhoram a performance ambiental das edificações.

Uma prática de ecodesign relatada por todas as empresas é o desenvolvimento de formulações da massa que podem incorporar resíduos sólidos gerados na conformação da massa, na estação de tratamento de efluentes líquidos industriais e nos filtros para mitigação da emissão do poluente fluoreto. 
A maioria das empresas já oferecem revestimentos de menor espessura. Esta solução de ecodesign não compromete a qualidade do produto, reduz o uso de matéria-prima e embalagem e seus custos de aquisição, diminui a emissão de fluoreto e $\mathrm{CO}_{2}$ por transporte. De fato, a redução de espessura é uma tendência mundial e tem potencial de reduzir até $50 \%$ o consumo de matérias-primas e decrescer a demanda de energia e água necessários para a manufatura, embalagem, transporte e armazenagem (GABALDÓN-ESTEVAN; CRIADO; MONFORT, 2014). No cluster de Criciúma, as empresas têm concentrado esforços em ecodesign voltado para produtos que gerem melhorias de performance ambiental de prédios.

O desenvolvimento dos programas de rotulagem no cluster paulista está baseado em parcerias com instituições ligadas ao setor de revestimentos cerâmicos, tais como, ASPACER, ANFACER, CCB e CETESB.

Todas as organizações usam embalagens fechadas nas laterais e com abertura no centro, nas faces frontal e traseira. Esta prática de eco-design possibilita a redução na quantidade de papelão, custo da embalagem e quantidade do resíduo gerado.

\subsection{Práticas de ecoinovação organizacional}

Após a implantação das ecoinovações organizacionais os fabricantes evidenciaram o alcance dos seguintes resultados: reaproveitamento de resíduos sólidos por organizações externas; prevenção de impactos ambientais na destinação ambientalmente adequada de resíduos sólidos; redução dos desperdícios de produto acabado; redução no consumo de energia elétrica; redução da emissão de material particulado; conscientização dos profissionais; e, gestão do desempenho ambiental. Esta compatibilidade de resultados mostra que os produtores de ambos os clusters mobilizam, de maneira similar, suas estruturas organizacionais (ex.: profissionais, equipes interdisciplinares, documentação, métodos de gestão e infraestrutura predial) na direção da sustentabilidade.

A contratação de empresas especializadas na destinação final ambientalmente adequada dos resíduos como restos de madeira de pallets, plástico, papelão, óleo e lâmpadas é amplamente difundida em ambos os clusters. Somente em algumas empresas do cluster de Santa Gertrudes há a contratação de empresas para fazer a destinação final adequada da cal fluorada, lodo e caco queimado. Todavia, existem empresas em ambos os clusters que fazem logística reversa de revestimentos para olarias.

Também vale destacar a participação de alguns produtores cerâmicos em programas de retorno de resíduos dos fornecedores, como o caso dos pallets, embalagens de tintas de impressora, fitas adesivas e embalagens.

As empresas também estabelecem parcerias com o varejo e construtoras para programas de retorno de produtos acabados. Esta é uma ação de logística reversa que previne o descarte inadequado do produto, reduz a perda de receita que poderia ser gerada por produtos não vendidos e evita a perda de credibilidade da marca.

Nos produtores brasileiros, a atualização dos sistemas de iluminação é uma iniciativa consolidada com evidência de implantação nas últimas duas décadas. Os resultados corroboram com o argumento de que a atualização dos sistemas de iluminação reduz o consumo de energia elétrica em $10 \%$. Outra prática é o uso de luz natural.

Por força de lei, em São Paulo, os caminhões devem ser cobertos carregados de argila ou não. Em Santa Catarina, a exigência é estar coberto somente quando carregado de argila. Há também iniciativas de aplicação de asfalto e umidificação de vias não pavimentadas para diminuir a emissão de material particulado.

Em ambos os clusters verificou-se a instalação de galpão coberto para o armazenamento das matérias-primas com o objetivo de reduzir a emissão atmosférica do material particulado que se desprende das matérias-primas armazenadas. Somente no cluster de Santa Gertrudes, esta ação é uma exigência da regulamentação. Estas ecoinovações contribuem para a diminuição dos problemas ambientais relacionados à concentração de material 
particulado na atmosfera da região dos clusters cerâmicos. A região de Santa Gertrudes detém uma das maiores concentrações de poluentes atmosféricos do estado de São Paulo.

Algumas organizações desenvolvem treinamentos ambientais formais. O tema comum em todos os treinamentos é a gestão de resíduos sólidos. Algumas empresas consultadas pretendem implantar treinamentos ambientais formais.

Uma prática comum nas empresas é o acumulo de funções tradicionais do cargo com as funções ambientais, apenas com algumas poucas exceções.

A implantação de comitês ambientais não é uma prática comum nas empresas, sendo que, poucas exceções foram evidenciadas.

A maioria das empresas do cluster de Santa Gertrudes declararam ter, em suas metas de médio prazo, a implantação do SGA baseado na ISO 14001. O consenso no cluster de Santa Gertrudes é que o estabelecimento de sistemas de gestão ambiental certificados pela ISO pode ajudar as empresas a aumentarem as vendas. Por outro lado, em Criciúma, as empresas relataram que a certificação ISO 14001 não leva a um aumento no número de clientes.

Poucas empresas integram as questões ambientais nas suas políticas institucionais.

\subsection{Aplicação do modelo teórico}

A Figura 2 apresenta a aplicação do modelo teórico a partir dos resultados empíricos.As práticas de ecoinovação de processo encontradas nos clusters são produção mais limpa, ecoeficiência, tecnologia de fim de tubo e 6R's. Conforme Penteado, Carvalho e Lintz (2016) cada vez mais haverá legislações mais severas para a indústria cerâmica. Deste modo, a implantação dessas práticas permite uma antecipação a elas. Bribián, Capilla e Usón (2011) destacam que a redução dos impactos ambientais causados pela atividade é relacionada a melhorias tecnológicas de processo. As práticas de ecoinovação de produto são o ecodesign, avaliação do ciclo de vida, gestão sustentável de materiais e programas de rotulagem ecológica. De acordo com Rodrigues, Pigosso e McAloone (2016), o desenvolvimento de produtos com melhor desempenho ambiental é um compromisso das empresas com o desenvolvimento sustentável. Não houve relatos de práticas de ecoinovação de produto com base nas normas da série ISO 14000. Isto pode ocorrer devido aos custos de certificação que são amplamente reconhecidos como barreiras de implantação dentro das organizações (SINGH; BRUECKNER; PADHY, 2015). Outro fato que pode influenciar a decisão dos gestores em não adotar tais normas é a falta de perspectiva que elas possam agregar valor ao produto. 


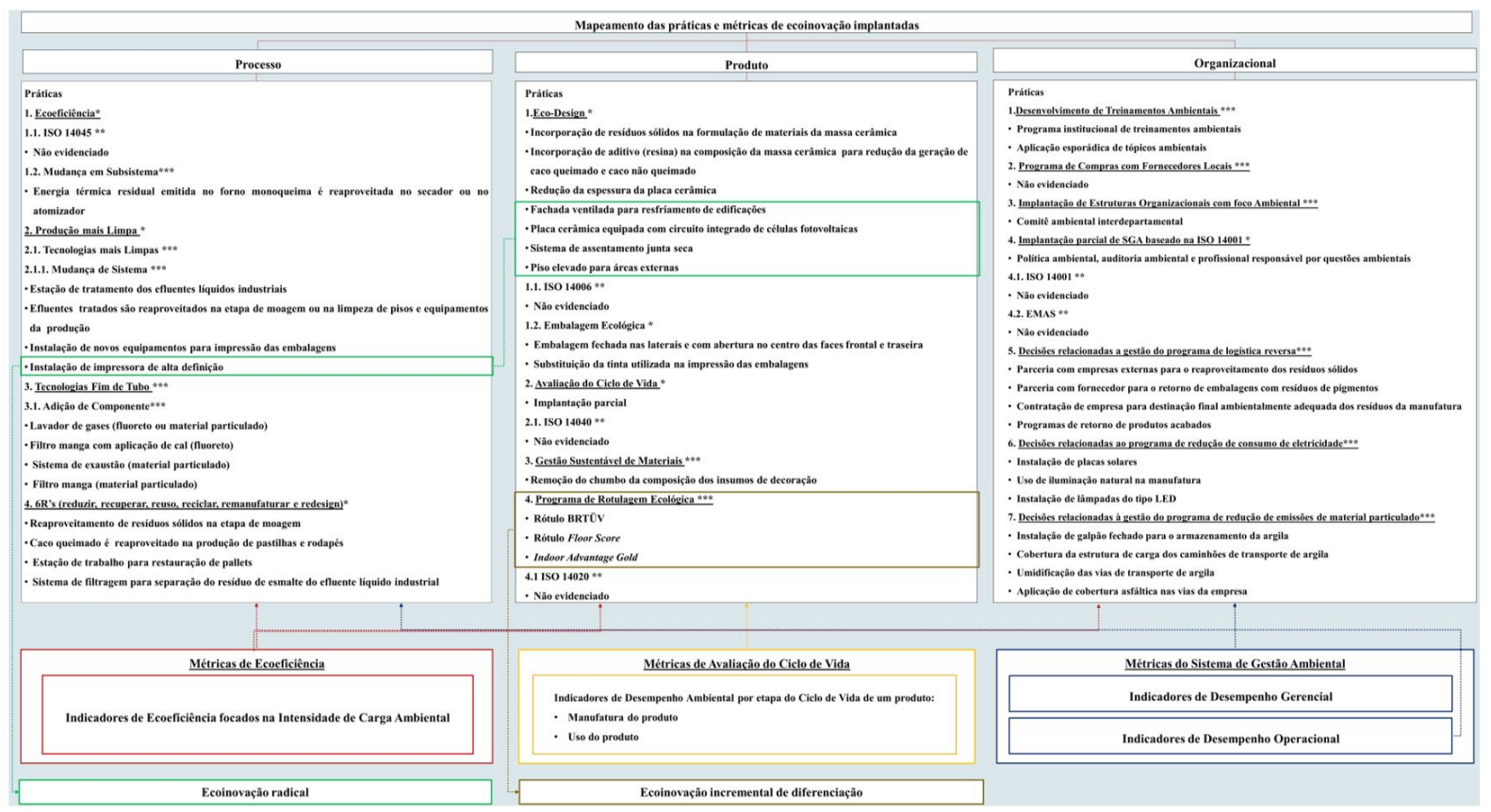

Fig.2. Aplicação do modelo teórico

Em relação às práticas de ecoinovação organizacional, destacam-se o desenvolvimento de treinamentos ambientais; implantação de estruturas organizacionais com foco ambiental; implantação parcial de SGA baseado na ISO 14001; logística reversa; programa de redução de consumo elétrico; e, programa de redução da emissão de material particulado. A demanda por programas de compras com fornecedores locais não foi relatada, pois os principais fornecedores já estão instalados nas regiões dos clusters pesquisados. Na indústria espanhola, a argila é importada de outros países, isto aumenta a demanda de energia e a emissão de poluentes no transporte.

Algumas métricas são utilizadas para mensurar mais de um tipo de ecoinovação. As empresas utilizam os indicadores de ecoeficiência focados na intensidade de carga ambiental para mesurar algumas das práticas de ecoinovação de processo, de produto e organizacional. Verificou-se que os KPIs podem ser utilizados para mensurar os resultados, tanto de ecoinovações de processo, como de ecoinovações organizacionais.

Os indicadores de LCA são concentrados em apenas duas etapas do ciclo de vida: manufatura do produto e uso do produto. Isso mostra que os produtores ainda não consideram, no desenvolvimento de produtos, os indicadores de desempenho ambiental relacionados à extração de matéria-prima, produção de material, fim de vida e transporte. Um fato que pode explicar a concentração de indicadores na etapa de manufatura é que significativa parte das regulamentações públicas são relacionadas à requerimentos focados na melhoria da performance ambiental dos processos produtivos. A incidência dos indicadores voltados à etapa de uso pode ser relacionada ao fato que estes indicadores ajudam os produtores a comunicarem aos clientes as melhorias que a aplicação de revestimentos pode gerar na performance ambiental das edificações.

A maioria das práticas de ecoinovação implantadas nos processos, produtos e métodos organizacionais são de cunho incremental e a principal motivação é reação ou antecipação a legislações ambientais ou demandas do mercado. Uma exceção é a implantação do rótulo ecológico que pode ser um fator de diferenciação para consumidores que consideram a responsabilidade ambiental como critério para a decisão de compra. Conforme Klewitz e Hansen (2014), esta prática pode ser definida como inovação incremental de diferenciação. Almeida et al. (2016) destaca a rotulagem ambiental como uma das iniciativas para melhorar o perfil ambiental da indústria portuguesa. 
Gabaldón-Estevan, Criado e Monfort (2014) indicam que as tecnologias incrementais implantadas na indústria espanhola não atendem os desafios da redução de consumo de energia e materiais. Nos produtores brasileiros parte das ecoinovações incrementais são endereçadas à redução do consumo desses recursos.

A tabela 1 descreve os resultados e impactos de cada prática de ecoinovação identificada.

Tabela 1. Resultados e impactos de cada prática de ecoinovação identificada

\begin{tabular}{|c|c|c|}
\hline Práticas de ecoinovação & $\begin{array}{l}\text { Tipo de } \\
\text { ecoinovação }\end{array}$ & Resultados e impactos \\
\hline $\begin{array}{l}\text { Energia térmica residual emitida no forno monoqueima é } \\
\text { reaproveitada no secador ou no atomizador }\end{array}$ & Processo & $\begin{array}{l}\text { Redução no consumo de gás natural } \\
\text { e emissão de fluoreto }\end{array}$ \\
\hline Estação de tratamento dos efluentes líquidos industriais & Processo & $\begin{array}{c}\text { Prevenção do descarte inadequado } \\
\text { de efluentes líquidos industriais não } \\
\text { tratados }\end{array}$ \\
\hline $\begin{array}{l}\text { Efluentes tratados são reaproveitados na etapa de moagem ou na } \\
\text { limpeza de pisos e equipamentos da produção }\end{array}$ & Processo & \multirow{2}{*}{$\begin{array}{l}\text { Redução no consumo de água de } \\
\text { poço artesiano ou fornecimento } \\
\text { externo }\end{array}$} \\
\hline $\begin{array}{l}\text { Piso elevado para áreas externas (para coletar e armazerar água } \\
\text { proveniente de chuva) }\end{array}$ & Produto & \\
\hline $\begin{array}{c}\text { Instalação de novos equipamentos para impressão das } \\
\text { embalagens }\end{array}$ & Processo & \multirow{2}{*}{$\begin{array}{c}\text { Redução no consumo de tinta } \\
\text { utilizada na impressão das } \\
\text { embalagens }\end{array}$} \\
\hline Substituição da tinta utilizada na impressão das embalagens & Produto & \\
\hline Instalação de impressora de alta definição & Processo & $\begin{array}{l}\text { Redução no consumo de pigmentos } \\
\text { e matéria-prima }\end{array}$ \\
\hline Lavador de gases & Processo & $\begin{array}{l}\text { Redução na emissão de material } \\
\text { particulado ou de fluoreto }\end{array}$ \\
\hline Filtro manga com aplicação de cal & Processo & Mitigação da emissão de fluoreto \\
\hline Sistema de exaustão & Processo & \multirow{6}{*}{$\begin{array}{l}\text { Redução da emissão de material } \\
\text { particulado }\end{array}$} \\
\hline Filtro manga & Processo & \\
\hline Instalação de galpão fechado para o armazenamento da argila & Organizacional & \\
\hline $\begin{array}{l}\text { Cobertura da estrutura de carga dos caminhões de transporte de } \\
\text { argila }\end{array}$ & Organizacional & \\
\hline Umidificação das vias de transporte de argila & Organizacional & \\
\hline Aplicação de cobertura asfáltica nas vias da empresa & Organizacional & \\
\hline $\begin{array}{l}\text { Reaproveitamento de resíduos sólidos na etapa de moagem } \\
\text { (cal florada, lodo, caco queimado e material particulado) }\end{array}$ & Processo & $\begin{array}{l}\text { Reutilização adequada de resíduos } \\
\text { sólidos }\end{array}$ \\
\hline $\begin{array}{l}\text { Caco queimado é reaproveitado na produção de pastilhas e } \\
\text { rodapés }\end{array}$ & Processo & \multirow[t]{2}{*}{$\begin{array}{l}\text { Redução no desperdício de matéria- } \\
\text { prima }\end{array}$} \\
\hline Incorporação de cacos não queimados na massa cerâmica & Processo & \\
\hline Estação de trabalho para restauração de pallets & Processo & $\begin{array}{l}\text { Redução do consumo e do } \\
\text { desperdício de pallets }\end{array}$ \\
\hline $\begin{array}{l}\text { Sistema de filtragem para separação do resíduo de esmalte do } \\
\text { efluente líquido industrial }\end{array}$ & Processo & $\begin{array}{l}\text { Redução do consumo e do } \\
\text { despedício de esmaltes }\end{array}$ \\
\hline Programas de treinamento ambiental (formais e informais) & Organizacional & $\begin{array}{l}\text { Conscientização da equipe de } \\
\text { colaboradores }\end{array}$ \\
\hline Comitê ambiental interdepartamental & Organizacional & Gestão da performance ambiental \\
\hline
\end{tabular}


Tabela 1. Continuação...

\begin{tabular}{|c|c|c|}
\hline Práticas de ecoinovação & $\begin{array}{l}\text { Tipo de } \\
\text { ecoinovação }\end{array}$ & Resultados e impactos \\
\hline $\begin{array}{l}\text { Política ambiental, auditoria ambiental e profissional responsável } \\
\text { por questões ambientais }\end{array}$ & Organizacional & \\
\hline $\begin{array}{c}\text { Parceria com empresas externas para o reaproveitamento dos } \\
\text { resíduos sólidos }\end{array}$ & Organizacional & $\begin{array}{l}\text { Reuso de resíduos sólidos por } \\
\text { organizações parceiras }\end{array}$ \\
\hline $\begin{array}{l}\text { Contratação de empresa para destinação final ambientalmente } \\
\text { adequada dos resíduos da manufatura (embalagens, substâncias } \\
\text { oleósas, pallets e cacos queimado e não queimado) }\end{array}$ & Organizacional & $\begin{array}{l}\text { Prevenção de impactos ambientais } \\
\text { com a disposição adequada dos } \\
\text { resíduos }\end{array}$ \\
\hline $\begin{array}{c}\text { Programas de retorno de produtos acabados (varejistas e } \\
\text { construtores) }\end{array}$ & Organizacional & $\begin{array}{l}\text { Reutilização dos resíduos e } \\
\text { prevenção de descarte inadequado }\end{array}$ \\
\hline Instalação de placas solares & Organizacional & \\
\hline Uso de iluminação natural nas unidades de fabricação & Organizacional & \\
\hline Atulalização dos sistemas de iluminação (LED) & Organizacional & $\begin{array}{c}\text { Redução no consumo de energia } \\
\text { elétrica }\end{array}$ \\
\hline $\begin{array}{l}\text { Placa cerâmica equipada com circuito integrado de células } \\
\text { fotovoltaicas }\end{array}$ & Produto & \\
\hline $\begin{array}{l}\text { Incorporação de resíduos sólidos na formulação de materiais da } \\
\text { massa cerâmica }\end{array}$ & Produto & Reuso de resíduos sólidos \\
\hline $\begin{array}{c}\text { Incorporação de aditivo (resina) na composição da massa } \\
\text { cerâmica para redução da geração de caco queimado e caco não } \\
\text { queimado }\end{array}$ & Produto & $\begin{array}{l}\text { Redução na geração de resíduos } \\
\text { sólidos }\end{array}$ \\
\hline Redução da espessura da placa cerâmica & Produto & $\begin{array}{l}\text { Redução no consumo de matéria- } \\
\text { prima, água e energias }\end{array}$ \\
\hline Fachada ventilada para resfriamento de edificações & Produto & $\begin{array}{l}\text { Redução da energia demandada } \\
\text { para a refrigeração artificial de } \\
\text { edificações }(30 \% \text { a } 50 \%)\end{array}$ \\
\hline Sistema de assentamento junta seca & Produto & $\begin{array}{l}\text { Eliminação da necessidade do } \\
\text { consumo de recursos naturais para a } \\
\text { produção do material rejunte. }\end{array}$ \\
\hline $\begin{array}{l}\text { Embalagem fechada nas laterais e com abertura no centro das } \\
\text { faces frontal e traseira }\end{array}$ & Produto & Redução no consumo de papelão \\
\hline Remoção do chumbo da composição dos insumos de decoração & Produto & $\begin{array}{l}\text { Eliminação de substâncias tóxicas } \\
\text { do produto acabado }\end{array}$ \\
\hline Programa de Rotulagem Ecológica & Produto & $\begin{array}{l}\text { Comunicação dos atributos } \\
\text { ambientais do produto acabado }\end{array}$ \\
\hline Nanotecnolia nas placas cerâmicas & Produto & $\begin{array}{l}\text { Redução na concentração de NOx } \\
\text { (óxido de nitrogênio) }\end{array}$ \\
\hline
\end{tabular}

\section{Conclusão}

A ecoinovação é um campo de pesquisa recente com os primeiros artigos publicados na década passada. A discussão sobre este tipo de inovação que combina benefícios ambientais e econômicos, tem ganhado nos últimos dez anos, crescente relevância nos meios acadêmico e profissional.

As práticas mais comuns implantadas em processo são a produção mais limpa, ecoeficiência, tecnologia de fim de tubo e 6R's. Em relação à ecoinovação de produto, as práticas mais implantadas são o ecodesign, avaliação do ciclo de vida, gestão sustentável de materiais e programas de rotulagem ecológica. No caso da 
ecoinovação organizacional, as práticas comumente implantadas são os treinamentos ambientais, estruturas organizacionais com foco ambiental, implantação parcial de SGA baseado na ISO 14001, logística reversa, programas de redução de consumo elétrico e de emissão de material particulado.

Também foram identificadas as métricas de ecoinovação. Observou-se que as métricas de ecoeficiência são aplicadas para mensurar as ecoinovações de processo, produto e organizacional. As métricas de avaliação de ciclo de vida são utilizadas somente para medir os resultados das ecoinovações de produto. Já as métricas do sistema de gestão ambiental podem ser aplicadas para aferir os resultados das práticas de ecoinovação de processo e organizacional.

Em relação as implicações gerenciais, espera-se que esse estudo possa ajudar à tomada de decisão considerando a implementação de ecoinovações na cadeia brasileira de revestimentos cerâmicos.

\section{Agradecimentos}

Os autores agradecem o suporte da Fapesp e da Capes.

\section{Referências}

ALMEIDA. M. I. et.al. Environmental profile of ceramic tiles and their potential for improvement. Journal of Cleaner Production, v.131, p.583-593, 2016. Disponível em: <http://dx.doi.org/10.1016/j.clepro.2016.04.131>. Acesso em: 27. Nov. 2017.

ARAMPATZIS, G. et al. A web-based Toolbox to support the systemic eco-efficiency assessment in water use systems. Journal of Cleaner Production, v. 138, p.181-194, Dec. 2016. Disponível em: <http://dx.doi.org/10.1016/j.jclepro.2016.02.065>. Acesso em: 10 jan. 2017.

ARENA, M.; AZZONE, G.; CONTE, A. A streamlined LCA framework to support early decision making in vehicle development. Journal of Cleaner Production, v. 41, p.105-113, Feb. 2013. Disponível em: <http://dx.doi.org/10.1016/j.jclepro.2012.09.031>. Acesso em: 13 jan. 2017.

BENVENISTE, G. et al. Análise do Ciclo de Vida Setorial de Placas Cerâmicas. Cerâmica Industrial, v. 17, n. 1, p. 7-13, jan./fev. 2012. Disponível em: <http://dx.doi.org/10.4322/cerind.2014.001>. Acesso em: 14 dez. 2017.

BOSSLE, M. B. et al.. The drivers for adoption of eco-innovation. Journal of Cleaner Production, v. 113, n. 1, p. 861-872, Feb. 2016. Disponivel em < http://dx.doi.org/10.1016/j.jclepro.2015.11.033> Acesso em: 15 ago. 2016.

BRIBIÁN, I. Z.; CAPILLA, A. V.; USÓN, A. A. Life cycle assessment of building materials: Comparative analysis of energy and environmental impacts and evaluation of the eco-efficiency improvement potential. Building And Environment, v. 46, n. 5 , p.1133-1140, maio 2011. Disponível em: <https://doi.org/10.1016/j.buildenv.2010.12.002>. Acesso em: 12 nov. 2017.

Brones, F., Carvalho, M.M., 2015. From 50 to 1: integrating literature toward a systemic ecodesign model. Journal of Cleaner Production, v. 96, p. 44-57. <https://doi.org/10.1016/j.jclepro.2014.07.036.> Acesso em: 05 jan. 2017.

CAMPOS, L. M. et al. Environmental performance indicators: a study on ISO 14001 certified companies. Journal of Cleaner Production, v. 99, p.286-296, July. 2015. Disponível em: <http://dx.doi.org/10.1016/j.jclepro.2015.03.019>. Acesso em: 19 jan. 2017.

CARRILLO-HERMOSILLA, J.; RÍO, P. del; KÖNNÖLÄ, T. Diversity of eco-innovations: Reflections from selected case studies. Journal of cleaner production, v. 18, n. 10-11, p.1073-1083, July. Disponível em: <doi:10.1016/j.jclepro.2010.02.014>. Acesso em: 10 abr. 2016.

CETESB. Guia técnico ambiental da indústria de cerâmica branca e de revestimento - série P+L. 2008. Disponível em <http://www.redeaplmineral.org.br/biblioteca/ceramica-de-revestimento/ceramica.pdf/view > Acesso em: 15 dez. 2016.

CHENG, C.; SHIU, E. Validation of a proposed instrument for measuring eco-innovation:An implementation perspective. Technovation, v. 32, p. 329-344, n. 6, Mar. 2012. Disponível em < doi: 10.1016/j.technovation.2012.02.001> Acesso em: 20 jun. 2016.

CPRH. O setor ceramista e o meio ambiente: guia prático para o licenciamento ambiental. 2010. Disponível em $<$ http://www.cprh.pe.gov.br/ARQUIVOS_ANEXO/cartilha\%20caramistas;0419;20101123.pZf> Acesso em: 14 dez. 2016.

DEMIREL, P.; KESIDOU, E. Stimulating different types of eco-innovation in the UK: Government policies and firm motivations. Ecological Economics, v. 70, n. 8, p. 1546-1557, June. 2011. Disponível em: <http://dx.doi.org/10.1016/j.ecolecon.2011.03.019>. Acesso em: 16 dez. 2016.

DÍAZ-GARCÍA, C.; GONZÁLEZ-MORENO, Á.; SÁEZ-MARTÍNEZ, F. Eco-innovation: insights from a literature review. Innovation: Management, Policy \& Practice, v. 17, p. 6-23, Apr. 2015. Disponível em < DOI:10.1080/14479338.2015.1011060> Acesso em: 02 abr. 2016.

EISENHARDT, K. M. Building Theories from Case Study Research. The Academy of Management Review, v. 14, p.532-550, Oct. 1989. Disponível em: <https://www.jstor.org/stable/258557>. Acesso em: 17 fev. 2017. 
FAULKNER, W.; BADURDEEN, F. Sustainable Value Stream Mapping (Sus-VSM): methodology to visualize and assess manufacturing sustainability performance. Journal of Cleaner Production, v. 85, p.8-18, Dez. 2014. Disponível em: <http://dx.doi.org/10.1016/j.jclepro.2014.05.042>. Acesso em: 05 jan. 2017.

GABALDÓN-ESTEVAN, D.; CRIADO, E.; MONFORT, E.. The green factor in European manufacturing: a case study of the Spanish ceramic tile industry. Journal Of Cleaner Production, v. 70, p.242-250, May 2014. Disponível em: <dx.doi.org/10.1016/j.jclepro.2014.02.018>. Acesso em: 27 nov. 2017.

JACQUEMIN, L; PONTALIER, P; SABLAYROLLES, C. Life cycle assessment (LCA) applied to the process industry: a review. The International Journal of Life Cycle Assessment, v. 17, n. 8, p.1028-1041, 3 may 2012. Disponível em: $<10.1007 / \mathrm{s} 11367-012-0432-9>$. Acesso em: 13 set. 2016.

JIMÉNEZ-GUERRERO, J. F.; GÁZQUEZ-ABAD, J. C.; CEBALLOS-SANTAMARÍA, G. Innovation in eco-packaging in private labels. Innovation, v. 17, n. 1, p.81-90, 2 Jan. 2015. Disponível em: <http://dx.doi.org/10.1080/14479338.2015.1011055>. Acesso em: 28 out. 2016.

KLEWITZ, J.; HANSEN, E. G. Sustainability-oriented innovation of SMEs: a systematic review. Journal of Cleaner Production, v. 65, p. 57-75, Feb. 2014. Disponível em <http://dx.doi.org/10.1016/j.jclepro.2013.07.017 > Acesso em: 10 nov. 2016.

LAOSIRIHONGTHONG, T.; ADEBANJO, D.; TAN, K. C. Green supply chain management practices and performance. Industrial Management \& Data Systems, v. 113, n. 8, p.1088-1109, 23 Aug. 2013. Disponível em: <DOI 10.1108/IMDS04-2013-0164>. Acesso em: 28 out. 2016.

LEVIDOW, L. et al. Process eco-innovation: assessing meso-level eco-efficiency in industrial water-service systems. Journal of Cleaner Production, v. 110, p. 54-65, Jan. 2016. Disponível em: <http://dx.doi.org/10.1016/j.jclepro.2014.12.086>. Acesso em: 10 jan. 2017.

LOZANO, R. Towards better embedding sustainability into companies' systems: an analysis of voluntary corporate initiatives. Journal of Cleaner Production, v. 25, p.14-26, Apr. 2012. Disponível em: <doi:10.1016/j.jclepro.2011.11.060>. Acesso em: 09 set. 2016.

NGUYEN, Q. A.; HENS, L. Environmental performance of the cement industry in Vietnam: the influence of ISO 14001 certification. Journal of Cleaner Production, v. 96, p.362-378, June. 2015. Disponível em: <http://dx.doi.org/10.1016/j.jclepro.2013.09.032>. Acesso em: 08 fev. 2017

ORGANISATION FOR ECONOMIC CO-OPERATION AND DEVELOPMENT (OECD). Eco-Innovation in Industry: Enabling Green Growth. 2009. Disponível em: <www.oecd.org/sti/ind/44424374.pdf>. Acesso em: 6. jan. 2017.

PENTEADO, C.; CARVALHO, E. V.; LINTZ, R. C. Reusing ceramic tile polishing waste in paving block manufacturing. Journal Of Cleaner Production, v. 112, p.514-520, Jan. 2016. Disponível em: <dx.doi.org/10.1016/j.jclepro.2015.06.142>. Acesso em: 26 nov. 2016.

POUDELET, V. et al. A process-based approach to operationalize life cycle assessment through the development of an ecodesign decision-support system. Journal of Cleaner Production, v. 33, p.192-201, Sep. 2012. Disponível em: <doi:10.1016/j.jclepro.2012.04.005>. Acesso em: 05 jan. 2016.

PRIETO-SANDOVAL, V. et al. ECO-labels as a multidimensional research topic: Trends and opportunities. Journal of Cleaner Production, v. 135, p.806-818, Nov. 2016. Disponível em: <http://dx.doi.org/10.1016/j.jclepro.2016.06.167>. Acesso em: 19 jan. 2017.

RODRIGUES. V. P, et.al. Process-related key performance indicators for measuring sustainability performance of eco-design implementation into product development. Journal of Cleaner Production, v. 139, p. 416-428, 2016. Disponível em:< http://dx.doi.org/10.1016/j.jclepro. 2016.08.046> Acesso em: 29. Nov.2017.

ROSCOE, S.; COUSINS, P. D.; LAMMING, R. C. Developing eco-innovations: a three-stage typology of supply networks. Journal of Cleaner Production, v. 112, n. 3, p. 1946-1959, July. 2015. Disponível em <doi:10.1016/j.jclepro.2015.06.125> Acesso em: 26 fev. 2016.

SECCHI, M. et al. Assessing eco-innovations in green chemistry: Life Cycle Assessment (LCA) of a cosmetic product with a biobased ingredient. Journal of Cleaner Production, v. 129, p.269-281, Aug. 2016. Disponível em: <http://dx.doi.org/10.1016/j.jclepro.2016.04.073>. Acesso em: 04 jan. 2017.

SINGH. M., et.al. Environmental management system ISO 14001: effective waste minimisation in small and medium enterprises in India. Journal of Cleaner Production, v.102, p.285-301, 2015. Disponível em: <http://dx.doi.org/10.1016/j.jclepro.2015.04.028> Acesso em: 29. Nov. 2017.

STANCHEV, P.; RIBAROVA, I. Complexity, assumptions and solutions for eco-efficiency assessment of urban water systems. Journal of Cleaner Production, v. 138, p.229-236, Dec. 2016. Disponível em: <http://dx.doi.org/10.1016/j.jclepro.2016.03.113>. Acesso em: 12 jan. 2017.

TRIGUERO, A.; MORENO-MONDÉJAR, L.; DAVIA, M. A. Drivers of different types of eco-innovation in European SMEs. Ecological Economics, v. 92, p. 25-33, Aug. 2013. Disponível em <doi:10.1016/j.ecolecon.2013.04.009> Acesso em: 04 jul. 2016. 